\title{
1,2-Cyclobutanecarboxylates Synthesized Using Photoreaction and Esterification
}

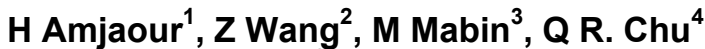 \\ ${ }^{1}$ University of North Dakota, Grand forks, ND, ${ }^{2} P h D$, Grand Forks, ND, ${ }^{3}$ Student, Grand Forks, ND, \\ ${ }^{4}$ advisor, Grand Forks, ND \\ houssein.amjaour@und.edu
}

Esters are importance targets or intermediates in the synthesis of plasticizers, perfumes, flavor chemicals, cosmetics, fine chemicals, pharmaceuticals, and chiral auxiliaries. However, ophthalates, a group of 1,2benzenedicarboxylates derived from petrochemicals, are being gradually replaced by phthalate-free plasticizers in many products due to health concerns. Truxinates, a family of 1,2-cyclobutanecarboxylates found in many natural products, share the structural similarities with o-phthalates and may serve as nontoxic phthalate alternatives. In this poster presentation, we report the synthesis of a series of mono- and di-esters of a 1,2-cyclobutanecarboxylic acid (CBDA-4, Figure 1) from trans-cinnamic acid using [2+2] photocycloaddition and subsequent esterification. The structures of many 1,2-cyclobutanecarboxylates were successfully determined by single crystal X-ray diffraction. These esters show promising potential for use in wide-ranging applications. For example, the cyclobutane ring in some esters was thermally cleavable at high temperature, providing a method by which materials made from them could be easily recyclable. References [1] Amjaour. H, Z. Wang, Mabin. M and Q. R. Chu, Chem. Commun. 2019, 55, 214-217. [2] Wang, Z.; Scheuring, M.; Mabin, M.; Shahni, R.; Wang, Z. D.; Ugrinov, A.; Butz, J.; Chu, Q. R., ACS Sustainable Chem. Eng. 2020, 8, 8909-8917. [3] B. Cornils and P. Lappe, "Dicarboxylic Acids, Aliphatic" in Ullmann's encyclopedia of industrial chemistry, Wiley-VCH, Weinheim, 2014.

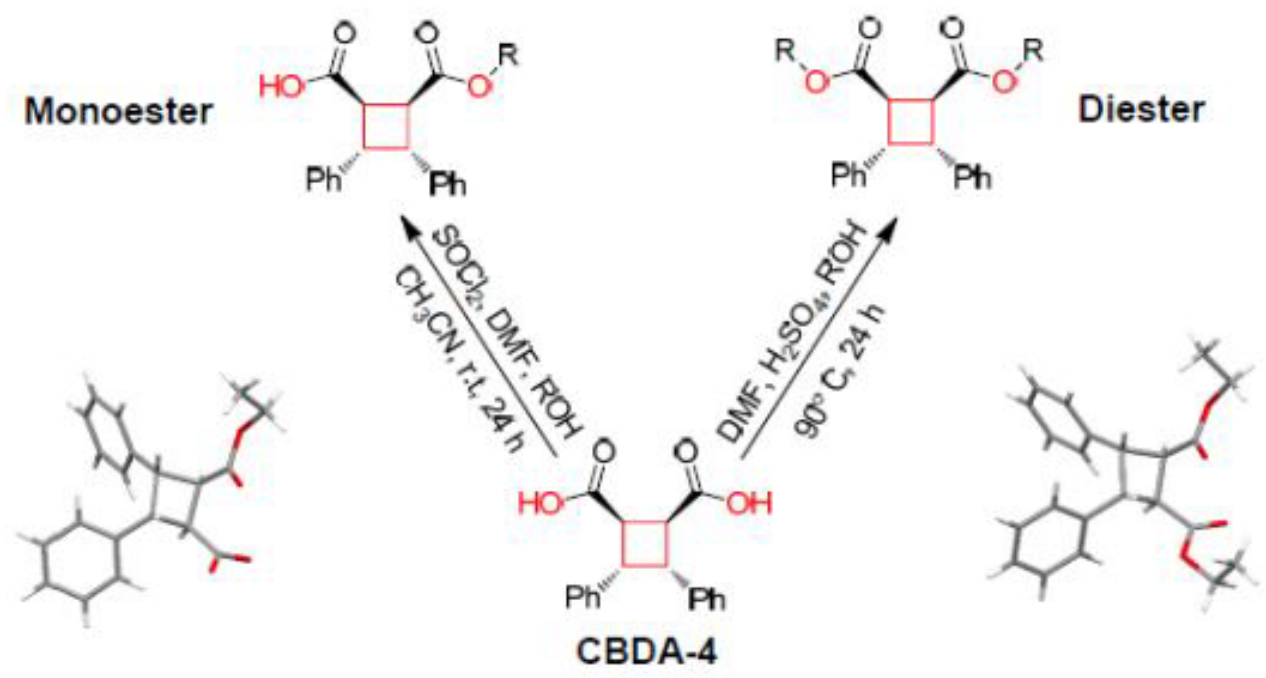

Figure 1. Synthesis of mono- and di-esters of CBDA-4 as well as the crystal structures of the mono- (left) and the di-(left) ethyl esters.

Acta Cryst. (2020). A76, a184 